\title{
Coronavirus disease 2019 (covid-19) and rheumatic diseases
}

At the end of December 2019, a new viral infection never before described appeared in the city of Wuhan, Hubei province, China, leading to a percentage of cases of severe respiratory failure. It was very quickly investigated and its etiological agent was discovered, being a new betacoronavirus, called Severe Acute Respiratory Coronavirus 2 Syndrome (SARS-Cov 2), to differentiate it from the SARS-Cov, reported in the period from 2000 to 2004, and another similar disease also caused by coronavirus, after ten years - Middle East Respiratory Syndrome Coronavirus (MERS-Cov). The disease caused by the virus came to be called Coronavirus Disease 2019 (Covid-19). After leaving China, this disease spread to several countries in the world, culminating in March, when the World Health Organization (WHO) declared a state of pandemic (1). The coronavirus is characterized by presenting RNA with a single helix and on its viral surface presents, to electron microscope, a structure that look like a crown, hence the name corona. A probable mutation happened from wild sources, which could be the origin of bats or snakes. Eating meat from the wild animal market, a tradition in China, was once again responsible for a major epidemic ${ }^{(1)}$.

More recent studies have shown that the virus uses the angiotensin-converting enzyme receptor (ECA2) to penetrate cells ${ }^{(2)}$. This receptor is abundant in the alveolar cells of the lungs, so this virus mainly affects these organs, in addition to the gastrointestinal tract, the heart and blood vessels ${ }^{(3)}$.

Apparently, all human beings are susceptible to acquire COVID-19, however the evolution to severe forms, characterizing the risk factors, was evaluated in the largest published study with more than 70,000 cases of this disease in China, generally occurring in elderly people over 60 years old and individuals who have comorbidities, such as systemic arterial hypertension, diabetes mellitus, heart disease, pneumopathies such as asthma and chronic obstructive pulmonary disease, and neoplasias. Interestingly, children does not seem risk group ${ }^{(4)}$. Other studies bring new data such as smoking, obesity, the male gender as additional risk factors to this already known risk constellation ${ }^{(5)}$.

Most patients with COVID-19 will have mild or moderate disease (81\%), then severe cases will be $14 \%$ and the dreaded critical cases will be $5 \%$. These individuals may need mechanical ventilation and have a high mortality rate in these cases, which can reach $80 \%$. Mortality in the general range varies from 1 to $3 \%{ }^{(6)}$.

Contagion occurs mainly through contamined droplets and direct contact between people. Thus, measures aimed at blocking droplets, such as the use of masks and hands cleaning are imperative in coping with this disease ${ }^{(7)}$.

Most people who contract this infection have a benign disease outcome; however, $15 \%$ require hospitalization, and $5 \%$ of these patients require intensive care. The latter group have a high rate of mortality, reaching 60 to $80 \%{ }^{(1)}$.

It is well known that patients with rheumatic disease (RD) are prone to infections, due to disease activity, use of immunosuppressive drugs, comorbidities and also immune dysfunctions. Therefore, there is current concern regarding whether RD patients are at increased risk for Covid-19. However, no such risk for RD patients has been detected until now; only a few patients with RD who died or had severe Covid-19 were described. In fact, an international consortium was established and until now 110 patients with rheuamtic disease were included. Rhuematoid arthrits was responsible for the majority (36\%), followed by lupus (17\%) and psoriatic arthritis (17\%), vasculitis in $7 \%$ and spondyloarthritis (7\%) and Sjögren syndrome in $5 \%$. Regarding comorbities, hypertension was observed in $28 \%$ of the cases, lung diseases in $20 \%$, cardiovascular disorders in $11 \%$, morbid obesity in $8 \%$ and diabetes in $8 \%$. From these 110 subjects, $35 \%$ were admitted to hospitals and $6(5 \%)$ unfortunately died ${ }^{(9)}$. Another artcile which described 5 cases of patients with rheumatic disease, in a total of 568 patients who looked for a Chinese hospital for COVID 19 , performing a small perecentage of $0.88 \%$ of the cases ${ }^{(10)}$. Four patients had rheumatoid arthritis and 1 
systemic sclerosis, which two had mild disease, 2 progressed to severe COVID and 1 needed critical care. All patients at the end had excelent evolution and were discharged ${ }^{(10)}$.

One reasonable hypothesis is that chloroquine or hydroxychloroquine, drugs commonly used for rheumatological conditions, might play a protective role. Another possibility is that the use of other immunomodulator drugs, common in these patients, may reduce or block inflammatory virus activity, or even prevent the cytokine storm sometimes seen in severe Covid-19, such as tocilizumab which inhibits interleukin-6 and anakinra (blocks interleukin 1). Chloroquine has antiviral properties against several viruses, including the SARS coronavirus, in in vitro studies (4); however, its clinical efficacy is still controversial ${ }^{(5)}$. Chloroquine has been included in guidelines from China, and clinical trials using this drug are ongoing ${ }^{(6)}$. More recently, two large studies with a total about 2,800 patients have shown no beneficial with this drug regarding mortality or intubation and mechanical ventilation rate ${ }^{(7,8)}$. Data ragarding antimalarials or other drugs in the early phase of the disease are needed.

Other drugs are also been tested in clinical trial for COVID, azithromycin is one of them, combined with chloroquine or used alone. In these two large observational studies described above ${ }^{(7,8)}$, this fact was also studied and, unfortunately, there were no beneficial effects of this antibiotic.

For now, it is essential to maintain preventive measures for all patients with RD, such as wearing masks, hands cleaning, isolation, quarantine, social distancing and lockdown, while maintaining use of immunomodulator drugs. Furthermore, close monitoring is essential while waiting for observational and prospective data on the incidence and clinical significance of COVID-19 in rheumatological subjects.

Vaccines or specific anti-viral drugs against COVID-19 are the great need at this moment in the world. Regarding to vaccination, some vaccine models for COVID-19 are already being identified and already tested in vitro, in animal models and even in humans. Let us hope that promising results come quickly.

Jozélio Freire de Carvalho

Institute for Health Sciences from Federal University of Bahia, Salvador-BA, Brazil

\section{REFERENCES}

1. WHO. Novel coronavirus (2019-nCoV) situation reports. Disponível em: https://www.who. int/emergencies/ diseases/novel-coronavirus-2019/situation-reports Acesso em: 31 Mar 2020.

2. GUAN, W.J. et al. Clinical characteristics of coronavirus disease 2019 in China. N Engl J. Med., Boston, 2020.

3. BHATRAJU, P. K. et al. Covid-19 in critically ill patients in the seattle region - case series. N. Engl. J. Med., Boston, 2020.

4. KEYAERTS, E. et al. In vitro inhibition of severe acute respiratory syndrome coronavirus by chloroquine. Biochem. Biophys Res. Commun., New York, v.323, p.264-268, 2004.

5. CORTEGIANI, A. et al. A systematic review on the efficacy and safety of chloroquine for the treatment of COVID-19. J. Crit. Care, Philadelphia, 2020.

6. ZHONGHUA, JIE HE HE HU XI ZA ZHI. Expert consensus on chloroquine phosphate for the treatment of novel coronavirus pneumonia. Biblioteca Nacional de medicina, [s.I], v. 43, p.185-188, 2020.

7. GELERIS, J. et al. Observational Study of Hydroxychloroquine in Hospitalized Patients with Covid-19. N. Engl. J. Med., Boston, 2020. DOI: 10.1056/NEJMoa2012410.

8. ROSENBERG, E. S. et al. Association of Treatment With hydroxychloroquine or Azithromycin With In-Hospital Mortality in Patients With COVID-19 in New York State. JAMA, London, 2020. DOI: 10.1001/jama.2020.8630.

9. GIANFRANCESCO, M.A.; HYRICH, K.L.; GOSSEC, L. Rheumatic disease and COVID-19: initial data from the COVID-19 Global Rheumatology Alliance provider registries. Lancet Rheumatol., [s.I], 2020. DOI: 10.1016/S26659913(20)30095-3.

10. CHENG, C. et al. COVID-19 with rheumatic diseases: a report of 5 cases. Clin. Rheumatol., Brussels, 2020. DOI: 10.1007/s10067-020-05160-x. 